\title{
THE NEAT EMBEDDING PROBLEM FOR ALGEBRAS OTHER THAN CYLINDRIC ALGEBRAS AND FOR INFINITE DIMENSIONS
}

\author{
ROBIN HIRSCH AND TAREK SAYED AHMED
}

\footnotetext{
Abstract. Hirsch and Hodkinson proved, for $3 \leq m<\omega$ and any $k<\omega$, that the class $S \mathrm{Nr}_{m} \mathbf{C A}_{m+k+1}$ is strictly contained in $S \mathrm{Nr}_{m} \mathbf{C A}_{m+k}$ and if $k \geq 1$ then the former class cannot be defined by any finite set of first order formulas, within the latter class. We generalise this result to the following algebras of $m$-ary relations for which the neat reduct operator $\mathfrak{N r}_{m}$ is meaningful: polyadic algebras with or without equality and substitution algebras. We also generalise this result to allow the case where $m$ is an infinite ordinal, using quasipolyadic algebras in place of polyadic algebras (with or without equality). ${ }^{1}$
}

Cylindric algebra is an algebraic correspondent of first-order logic with no constants or functions, more specifically $n$-dimensional cylindric algebra, $\mathbf{C A}_{n}$, is an algebraic correspondent of first-order logic restricted to $n$ indexed variables, for finite $n$. An algebra in $\mathbf{C A}_{n}$ is a boolean algebra together with a cylindrifier $c_{i}$, which acts as a unary operator and corresponds to existential quantification of the $i$ 'th variable, and a diagonal $\mathrm{d}_{i j}$ element corresponding to the equality of the $i$ th and $j$ 'th variable, for $i, j<n$. For $m<n$, the neat reduct $\mathfrak{N r}_{m} \mathcal{C}$ of a $\mathcal{C} \in \mathbf{C A}_{n}$ is the $m$-dimensional cylindric algebra obtained by restricting to those elements $c \in \mathcal{C}$ such that $\mathrm{c}_{i} c=c$ for $m \leq i<n$, and restricting to those cylindrifiers and diagonals indexed by $m$. If $\mathbf{K} \subseteq \mathbf{C A}_{n}$ we write $\mathbf{N r}_{m} \mathbf{K}$ for $\left\{\mathfrak{N r}_{m} \mathfrak{C}: \mathfrak{C} \in \mathbf{K}\right\}$. It is not the case that every algebra in $\mathbf{C A}_{m}$ is the neat reduct of an algebra in $\mathbf{C A}_{n}$, nor need it even be a subalgebra of a neat reduct of an algebra in $\mathbf{C A}_{n}$. Furthermore, $S \mathrm{Nr}_{m} \mathbf{C A}_{m+k+1} \neq S \mathrm{Nr}_{m} \mathbf{C A}_{m+k}$, whenever $3 \leq m<\omega$ and $k<\omega[10]$. A consequence of this is that there are $m$-variable formulas that can be proved with $m+k+1$-variables, but not with $m+k$-variables, in a certain, fairly typical, proof system.

Other algebras may be defined corresponding to restrictions or extensions of the $n$-variable first order logic described above. Because our focus is on neat reducts, we will only consider $n$-dimensional algebras where the cylindrifiers $\mathrm{c}_{i}$ are included, or at least are definable, within the set of operators of the algebra. Without that restriction it would not be possible to define a neat reduct and our algebras would correspond to first order logic without quantifiers, we do not consider that case here. But we might choose to drop the diagonals from our signature (corresponding to first order logic without equality), or we may add 
permutation operators, corresponding to permutations of the variables in first order logic. By generalising the results about neat reducts of $m$-dimensional cylindric algebras to other $m$-dimensional algebras, such as polyadic algebras with or without equality, diagonal free cylindric algebras and substitution algebras, one may deduce that there are $m$-variable formulas provable with $m+k+1$-variables but not with $m+k$-variables in logical proof systems similar to the one in [9], but with additional inference rules (corresponding to polyadic equality algebras (PEA)) or fewer weaker inference rules (corresponding to substitution algebras $(\mathbf{S c}))$.

Preliminaries. For cardinals $m, n$ we write ${ }^{m} n$ for the set of maps from $m$ to $n$. If $U$ is an ultrafilter over $\wp(I)$ and if $\mathcal{A}_{i}$ is some structure (for $i \in I$ ) we write either $\Pi_{i \in I} \mathcal{A}_{i} / U$ or $\Pi_{i / U} \mathcal{A}_{i}$ for the ultraproduct of the $\mathcal{A}_{i}$ over $U$. Fix some ordinal $n \geq 2$. For $i, j<n$ the replacement $[i / j]$ is the map that is like the identity on $n$ except that $i$ is mapped to $j$ and the transposition $[i, j]$ is the like the identity on $n$ except that $i$ is swapped with $j$. A map $\tau: n \rightarrow n$ is finitary if the set $\{i<n: \tau(i) \neq i\}$ is finite, so if $n$ is finite then all maps $n \rightarrow n$ are finitary. It is known, and not hard to show, that any finitary permutation is a product of transpositions and any finitary non-injective map is a product of replacements.

The standard reference for all the classes of algebras mentioned previously is [5]. Each class in $\left\{\mathbf{D f}_{n}, \mathbf{S c}_{n}, \mathbf{C A}_{n}, \mathbf{P A} \mathbf{A}_{n}, \mathbf{P E A} \mathbf{A}_{n}, \mathbf{Q P A} \mathbf{A}_{n}, \mathbf{Q P E A} \mathbf{A}_{n}\right\}$ consists of boolean algebras with extra operators, as shown in figure 1 , where $\mathrm{d}_{i j}$ is a nullary operator (constant), $\mathrm{c}_{i}, \mathrm{~s}_{\tau}, \mathrm{s}_{[i / j]}$ and $\mathrm{s}_{[i, j]}$ are unary operators, for $i, j<n, \tau$ : $n \rightarrow n$. For finite $n$, polyadic algebras are the same as quasi-polyadic algebra and for the infinite dimensional case we restrict our attention to quasi-polyadic algebras in $\mathbf{Q P A}_{n}, \mathbf{Q P E A} \mathbf{A}_{n}$. Each class is defined by a finite set of equation schema. Existing in a somewhat scattered form in the literature, equations defining $\mathbf{S c}_{n}, \mathbf{Q P A} \mathbf{A}_{n}$ and $\mathbf{Q P E A} \mathbf{A}_{n}$ are given in the appendix, definition 15. For $\mathbf{C A}_{n}$ we follow the standard axiomatization given in in [4, definiton 1.1.1]. For any operator $o$ of any of these signatures, we write $\operatorname{dim}(o)(\subseteq n)$ for the set of dimension ordinals used by $o$, e.g. $\operatorname{dim}\left(\mathrm{c}_{i}\right)=\{i\}, \operatorname{dim}\left(\mathrm{s}_{[i / j]}\right)=\operatorname{dim}\left(\mathrm{d}_{i j}\right)=$ $\{i, j\}$. An algebra $\mathcal{A}$ in $\mathbf{Q P E A}_{n}$ has operators that can define any operator of $\mathbf{Q P A} \mathbf{A}_{n}, \mathbf{C A} \mathbf{A}_{n}, \mathbf{S c}_{n}$ and $\mathbf{D f _ { n }}$. Thus we may obtain the reducts $\mathfrak{R \mathfrak { d } _ { K }}(\mathcal{A})$ for $K \in\left\{\mathbf{Q P E A}_{n}, \mathbf{Q P A} \mathbf{A}_{n}, \mathbf{C} \mathbf{A}_{n}, \mathbf{S c}_{n}, \mathbf{D f _ { n }}\right\}$ and it turns out that the reduct always satisfies the equations defining the relevant class so $\mathfrak{R d} \mathfrak{d}_{K}(\mathcal{A}) \in K$. Similarly from any algebra $\mathcal{A}$ in any of the classes $\mathbf{Q P E A} \mathbf{A}_{n}, \mathbf{Q P A} \mathbf{A}_{n}, \mathbf{C A}_{n}, \mathbf{S c}_{n}$ we may obtain the reduct $\mathfrak{R d _ { \mathbf { S c } }}(\mathcal{A}) \in \mathbf{S c}_{n}[2]$.

Let $\mathbf{K} \in\{\mathbf{Q P E A}, \mathbf{Q P A}, \mathbf{C A}, \mathbf{S c}, \mathbf{D f}\}$, let $\mathcal{A} \in K_{n}$ and let $2 \leq m \leq n$ (possibly infinite ordinals). The reduct to $m$ dimensions $\mathfrak{R d} \mathfrak{d}_{m}(\mathcal{A}) \in \mathbf{K}_{m}$ is obtained from $\mathcal{A}$ by restricting to those operators $o$ such that $\operatorname{dim}(o) \subseteq m$. The neat reduct to $m$ dimensions is the algebra $\mathfrak{N r}_{m}(\mathcal{A}) \in K_{m}$ with universe $\{a \in \mathcal{A}$ : $\left.m \leq i<n \rightarrow \mathrm{c}_{i} a=a\right\}$ where all operators $o$ with $\operatorname{dim}(o) \subseteq m$ are induced from $\mathcal{A}$ (see [4, definition 2.6.28] for the CA case). More generally, for $\Gamma \subseteq n$ we write $\mathfrak{N r}_{\Gamma} \mathfrak{A}$ for the algebra whose universe is $\left\{a \in \mathcal{A}: i \in n \backslash \Gamma \rightarrow \mathrm{c}_{i} a=a\right\}$ with all the operators $o$ of $\mathcal{A}$ where $\operatorname{dim}(o) \subseteq \Gamma$. Let $\mathcal{A} \in K_{m}, \mathcal{B} \in K_{n}$. An injective homomorphism $f: \mathcal{A} \rightarrow \mathcal{B}$ is a neat embedding if the range of $f$ is a 


\begin{tabular}{l|l} 
class & extra operators \\
\hline $\mathbf{D f}_{n}$ & $\mathrm{c}_{i}: i<n$ \\
$\mathbf{S c}_{n}$ & $\mathrm{c}_{i}, \mathrm{~s}_{[i / j]}: i, j<n$ \\
$\mathbf{C A}_{n}$ & $\mathrm{c}_{i}, \mathrm{~d}_{i j}: i, j<n$ \\
$\mathbf{P A}_{n}$ & $\mathrm{c}_{i}, \mathrm{~s}_{\tau}: i<n, \tau \in{ }^{n} n$ \\
$\mathbf{P E A}_{n}$ & $\mathrm{c}_{i}, \mathrm{~d}_{i j}, \mathrm{~s}_{\tau}: i, j<n, \tau \in{ }^{n} n$ \\
$\mathbf{Q P A}_{n}$ & $\mathrm{c}_{i}, \mathrm{~s}_{[i / j]}, \mathrm{s}_{[i, j]}: i, j<n$ \\
$\mathbf{Q P E A}_{n}$ & $\mathrm{c}_{i}, \mathrm{~d}_{i j}, \mathrm{~s}_{[i / j]}, \mathbf{s}_{[i, j]}: i, j<n$
\end{tabular}

Figure 1. Non-boolean operators for the classes

subalgebra of $\mathfrak{N r}_{m}(\mathcal{B})$. The notions of neat reducts and neat embeddings have proved useful in analyzing the number of variables needed in proofs, as well as for proving representability results, via the so-called neat embedding theorems $[1,11,12]$.

Let $m \leq n$ be ordinals and let $\rho: m \rightarrow n$ be an injection. For any $n$ dimensional algebra $\mathcal{B}$ (substitution, cylindric or quasi-polyadic algebra with or without equality) we define an $m$-dimensional algebra $\mathfrak{R d}^{\rho}(\mathcal{B})$, with the same universe and boolean structure as $\mathcal{B}$, where the $(i j)$ th diagonal of $\mathfrak{R d}^{\rho}(\mathcal{B})$ is $\mathrm{d}_{\rho(i) \rho(j)} \in \mathcal{B}$ (if diagonals are included in the signature of the algebra), the $i$ th cylindrifier is $\mathrm{c}_{\rho(i)}$, the $i$ for $j$ replacement operator is the operator $\mathrm{s}_{\rho(j)}^{\rho(i)}$ of $\mathcal{A}$ when it is not term definable from the other operations, namely, in the two cases of Sc and QPA (in the presence of diagonal elements and cylindrifiers these operations are term definable), the $i j$ transposition operator is $\mathbf{s}_{\rho(i) \rho(j)}$ if included in the signature, for $i, j<m$. It is easy to check, for $\mathbf{K} \in\{\mathbf{D f}, \mathbf{S c}, \mathbf{C A}, \mathbf{Q P A}, \mathbf{Q P E A}\}$, that if $\mathcal{B} \in \mathbf{K}_{n}$ then $\mathfrak{R d}^{\rho}(\mathcal{B}) \in \mathbf{K}_{m}$. Also, for $\mathcal{B} \in \mathbf{K}_{n}$ and $x \in \mathcal{B}$, we define $\mathfrak{R l}_{x}(\mathcal{B})$ by 'restriction to $x$ ', so the universe is the set of elements of $\mathcal{B}$ below $x$, where the boolean unit is $x$, boolean zero and sum are not changed, boolean complementation is relative to $x$, and the result of applying any non-boolean operator is obtained by using the operator for $\mathcal{B}$ and intersecting with $x$. It is not always the case that $\mathfrak{R l}_{x}(\mathcal{B})$ is a $\mathbf{K}_{n}$ (we can lose commutativity of cylindrifiers).

The main question we address in this paper is whether $S \mathrm{Nr}_{m} \mathbf{K}_{n}=\mathbf{K}_{m}$, where $m<n$ are possibly infinite ordinals and $\mathbf{K} \in\{\mathbf{D f}, \mathbf{S c}, \mathbf{C A}, \mathbf{Q P A}, \mathbf{Q P E A}\}$ and, if not, whether $S \mathrm{Nr}_{m} \mathbf{K}_{n}$ may be defined within $\mathbf{K}_{m}$ using only finitely many axioms (or finitely many axiom schemas, when $m$ is infinite). The case $\mathbf{K}=\mathbf{D f}$ of diagonal free algebra is easily answered: $S \mathrm{Nr}_{m} \mathbf{D \mathbf { f } _ { n }}=\mathbf{D f}_{m}$, for $3 \leq m \leq n$, see [5, theorem 5.1.31]. We show that in all the other cases, the answers are negative. In order to generalise the results of [10] to these other classes of algebra, we define an $m$-dimensional polyadic equality type algebra $\mathfrak{C}(m, n, r)$ where $3 \leq$ $m \leq n, r<\omega$ (see definition 4 below). These algebras are based on a relation algebra construction that first appeared in $[6,7]$ or see $[8$, section 15.2$]$, modified here so that the elements become $n$-dimensional rather than two dimensional. Still, although they are $n$-dimensional, all of their elements are generated by two dimensional elements. We will then prove the following theorem.

THEOREM 1. Let $3 \leq m \leq n$ and $r<\omega$. 
I. $\mathfrak{C}(m, n, r) \in \mathrm{Nr}_{m} \mathbf{Q P E A}_{n}$,

II. $\mathfrak{R} \mathfrak{O}_{\mathbf{S c}} \mathfrak{C}(m, n, r) \notin S \mathrm{Nr}_{m} \mathbf{S c}_{n+1}$,

III. $\Pi_{r / U} \mathfrak{C}(m, n, r)$ is elementarily equivalent to a countable polyadic equality algebra $\mathfrak{C} \in \mathrm{Nr}_{m}$ QPEA $\mathbf{A}_{n+1}$.

The proof of this theorem is the substantial part of this paper. The proofs are similar to proofs of corresponding results in [7] but modified for the signatures considered here and with some further modifications. To prove the first two parts, the algebras we consider will only have elements generated by two dimensional elements, however for the third part we will also consider elements that are essentially three dimensional (hence we will introduce three dimensional hypernetworks, for this part only). From this theorem we deduce the following.

COROLLARY 2. Let $\mathbf{K} \in\{$ QPEA, QPA, CA, Sc $\}$, let $3 \leq m<n<\omega$. Then $S \mathrm{Nr}_{m} \mathbf{K}_{n+1}$ is a proper subclass of $S \mathrm{Nr}_{m} \mathbf{K}_{n}$ which cannot be defined, within $S \mathrm{Nr}_{m} \mathbf{K}_{n+1}$, by any finite set of first order sentences.

Proof. We remarked earlier that for each choice of $\mathbf{K}$ and each $n$, the operators of $\mathbf{S} \mathbf{c}_{n}$ are definable by the operators of $\mathbf{K}_{n}$ which are themselves definable by the operators of $\mathbf{Q P E A} \mathbf{A}_{n}$. Hence, it follows from (I) that $\mathfrak{R} \mathfrak{d}_{\mathbf{K}} \mathfrak{C}(m, n, r) \in$ $\mathrm{Nr}_{m} \mathbf{K}_{n}$, from (II) $\mathfrak{R} \mathfrak{d}_{\mathbf{K}} \mathfrak{C}(m, n, r) \notin S N r_{m} \mathbf{K}_{n+1}$, for $r<\omega$ and from (III) $\mathfrak{R} \mathfrak{d}_{\mathbf{K}}(\mathfrak{C}) \in \mathrm{Nr}_{m} \mathbf{K}_{n+1}$. Now suppose for contradiction that $\phi$ is a sentence defining $S \mathrm{Nr}_{m} \mathbf{K}_{n}$ within $S \mathrm{Nr}_{m} \mathbf{K}_{n+1}$. Let $U$ be any non-principal ultrafilter over $\omega$. Since $\mathfrak{R} \mathfrak{d}_{\mathbf{K}} \mathfrak{C}(m, n, r) \in \mathrm{Nr}_{m} \mathbf{K}_{n} \backslash S \mathrm{Nr}_{m} \mathbf{K}_{n+1}, \mathfrak{R} \mathfrak{d}_{\mathbf{K}}(\mathfrak{C}(m, n, r)) \not \forall \phi$, for each $r<\omega$. By Łoś's theorem, $\Pi_{r / U} \mathfrak{R} \mathfrak{d}_{\mathbf{K}} \mathfrak{C}(m, n, r) \mid \neg \phi$. By elementary equivalence $\mathfrak{C} \models \neg \phi$, contradicting (III).

We will prove (I), (II), (III) below, after we have defined the algebras $\mathfrak{C}(m, n, r)$. For some time to come we restrict our attention to finite ordinals, which we denote by $m, n \ldots$, etc.

Main Construction. Now we define algebras $\mathfrak{C}(m, n, r) \in \mathbf{Q P E A}_{m}$ for $3 \leq$ $m \leq n<\omega$ and any linear order $r$. These algebras are based on the relation algebras defined in [6, section 3].

DEFINITION 3. Define a function $\kappa: \omega \times \omega \rightarrow \omega$ by $\kappa(x, 0)=0($ all $x<\omega)$ and $\kappa(x, y+1)=1+x \times \kappa(x, y)$ ) (all $x, y<\omega)$. For $n, r<\omega$ let

$$
\psi(n, r)=\kappa((n-1) r,(n-1) r)+1 .
$$

All of this is simply to ensure that $\psi(n, r)$ is sufficiently big compared to $n, r$ for the proof of non-embeddability to work. The second parameter $r<\omega$ may be considered as a finite linear order of length $r$. We may extend the definition of $\psi$ to the case where its second parameter is an arbitrary linear order by letting $\psi(n, r)=\omega$ for any infinite linear order $r$. For any $n<\omega$ and any linear order $r$, let

$$
\operatorname{Bin}(n, r)=\{I d\} \cup\left\{a^{k}(i, j): i<n-1, j \in r, k<\psi(n, r)\right\}
$$


where $I d, a^{k}(i, j)$ are distinct objects indexed by $k, i, j$. For $i<n-1, j \in r, k<$ $\psi(n, r)$ let

$$
\begin{aligned}
a(i,-) & \left.=\left\{a^{k}(i, j): j \in r, k<\psi(n, r)\right)\right\}, \\
a(-, j) & =\left\{a^{k}(i, j): i<n-1, k<\psi(n, r)\right\}, \\
a^{k} & =\left\{a^{k}(i, j): i<n-1, j \in r\right\}, \\
a(i, j) & =\left\{a^{k}(i, j): k<\psi(n, r)\right\}, \\
a(-,>j) & =\bigcup_{j<j^{\prime} \in r} a\left(-, j^{\prime}\right) \\
a(-, \leq j) & =\bigcup_{j^{\prime} \leq j \in r} a\left(-, j^{\prime}\right) \text { and } \\
a & =\bigcup_{i<n-1} a(i,-) .
\end{aligned}
$$

Let $3 \leq m \leq n<\omega$ and let $r$ be any linear order. Let $F(m, n, r)$ be the set of all functions $f: m \times m \rightarrow \operatorname{Bin}(n, r)$ such that for all $x, y, z<m$ we have $f(x, x)=I d, f(x, y)=f(y, x)$, and $(f(x, y), f(y, z), f(x, z)) \notin$ Forb, where Forb (the forbidden triples) is the following set of triples

$$
\begin{gathered}
\{(I d, b, c): b \neq c \in \operatorname{Bin}(n, r)\} \\
\cup \\
\left\{\left(a^{k}(i, j), a^{k^{\prime}}(i, j), a^{k^{*}}\left(i, j^{\prime}\right)\right): k, k^{\prime}, k^{*}<\psi(n, r), i<n-1, j^{\prime} \leq j \in r\right\} .
\end{gathered}
$$

Since the variables $x, y, z$ in the definition of $F(m, n, r)$ are universally quantified, it actually follows that $(f(x, y), f(y, z), f(x, z))$ avoids all Peirceans of forbidden triples, for $f \in F(m, n, r)$, e.g. we cannot have $(f(x, y), f(y, z), f(x, z))=$ $(b, I d, c)$ for $b \neq c$ since this would entail $(f(y, z), f(z, x), f(y, x))=(f(y, z), f(x, z), f(x, y))=$ $(I d, c, b) \notin$ Forb, contrary to the definition of Forb. For any $f, g \in F(m, n, r)$ and $x, y<m$ we write $f \equiv_{x y} g$ if for all $w, z \in m \backslash\{x, y\}$ we have $f(w, z)=g(w, z)$. We may write $f \equiv_{x} g$ instead of $f \equiv_{x x} g$. For $\tau: m \rightarrow m$ we write $(f \tau)$ for the function defined by

$$
(f \tau)(x, y)=f(\tau(x), \tau(y))
$$

Clearly, if $f \in F(m, n, r)$ then $(f \tau) \in F(m, n, r)$.

For the next couple of sections we will consider cases where $r<\omega$ is a finite linear order. The idea behind these algebras $\mathfrak{C}(m, n, r)$ (formalised below) may be sketched as follows. To prove theorem 1(II) we will assume for contradiction that $\mathfrak{R d _ { \mathbf { S c } }} \mathfrak{C}(m, n, r) \subseteq \mathfrak{N r C}$ for some $\mathfrak{C} \in \mathbf{S c}_{n+1}$, some finite $m, n, r$. We will show, by an inductive proof, that there must be a large set $S$ of distinct elements of $\mathfrak{C}$, satisfying certain inductive assumptions, which we outline next. For each $s \in S$ and $i, j<n+1$ there is an element $\alpha(s, i, j) \in \operatorname{Bin}(n, r)$ obtained from $s$ by cylindrifying all dimensions in $(n+1) \backslash\{i, j\}$, then using substitutions to replace $i, j$ by 0,1 . We show that $(\alpha(s, i, j), \alpha(s, j, k), \alpha(s, i, k)) \notin$ Forb, for all $s \in S$ and $i, j, k<n+1$. Our inductive assumptions state, among other things, that $\mathrm{c}_{n}(s)$ is constant, for $s \in S$, and for $l<n$ there are fixed $i<n-1, j<r$ such that for all $s \in S$ we have $\alpha(s, l, n) \leq a(i, j)$. This defines two functions $I: n \rightarrow(n-1), J: n \rightarrow r$ such that $\alpha(s, l, n) \leq a(I(l), J(l))$ for all $s \in S$. 
The rank $\rho(I, J)$ of $(I, J)$ is the sum (over $i<n-1$ ) of the maximum $j$ with $I(l)=i, J(l)=j$ (some $l<n)$ or -1 if there is no such $l$. We will prove that there is a set $S^{\prime}$ with index functions $\left(I^{\prime}, J^{\prime}\right)$, still relatively large (large in terms of the number of times we need to repeat the induction step) where the same induction hypotheses hold but where $\rho\left(I^{\prime}, J^{\prime}\right)>\rho(I, J)$. By repeating this enough times (more than $n r$ times) we obtain a non-empty set $T$ with index functions of rank strictly greater than $(n-1) \times(r-1)$, an impossibility.

We sketch the induction step. Since I cannot be injective there must be distinct $l_{1}, l_{2}<n$ such that $I\left(l_{1}\right)=I\left(l_{2}\right)$ and $J\left(l_{1}\right) \leq J\left(l_{2}\right)$. We may use $l_{1}$ as a "spare dimension" (changing the index functions on l will not reduce the rank). Since $\mathrm{c}_{n}(s)$ is constant, we may fix $s_{0} \in S$ so that for each $s \in S \backslash\left\{s_{0}\right\}$ we can pick $b \in \operatorname{Bin}(n, r)$ such that $s^{\prime}=\mathrm{c}_{l} s_{0} \cdot \mathrm{s}_{[n / l]} \mathrm{c}_{l} s \cdot \alpha(b, l, n)$ is non-zero, using the complete additivity of the operators. Let $S^{*}=\left\{s^{\prime}: s \in S \backslash\left\{s_{0}\right\}\right\}$, we wish to reestablish the induction hypotheses for $S^{*}$, and many of these are simple to check. But suitable functions $I^{\prime}, J^{\prime}$ might not exist because $\alpha(s, l, n) \in \operatorname{Bin}(n, r) \backslash\{I d\}$ might vary as $s$ ranges over $S \backslash\left\{s_{0}\right\}$ (for $l^{\prime} \neq l<n$ we can let $I^{\prime}\left(l^{\prime}\right)=I\left(l^{\prime}\right)$ and $\left.J^{\prime}\left(l^{\prime}\right)=J\left(l^{\prime}\right)\right)$. Still, because there are just $(n-1) r$ possible values for the $i, j$ indices of $\alpha(s, l, n)$ as $s$ ranges over $S \backslash s_{0}$ there must be a subset $S^{\prime} \subseteq S^{*}$ with $\left|S^{\prime}\right| \geq \frac{|S|-1}{n r}$ and where there exist $i<n-1, j<r$ such that for all $s \in S \backslash\left\{s_{0}\right\}$ we have $\alpha(s, l, n) \leq a(i, j)$. Now we let $I^{\prime}, J^{\prime}$ be identical to $I, J$ respectively, except $I^{\prime}(l)=i, J^{\prime}(l)=j$. With these index functions, the required set is $S^{\prime}$ and we check all the induction hypotheses. The size of $S^{\prime}$ is at least $\frac{|S|-1}{(n-1) r}$, still big enough to continue. It remains to show that the rank of $\left(I^{\prime}, J^{\prime}\right)$ is strictly greater than that of $(I, J)$. For this, we show that $J^{\prime}(l) \geq J(l)$ for all $l<n$. Since $(\alpha(s, i, j), \alpha(s, j, k), \alpha(i, k)) \notin$ Forb and by the definition of Forb either $\operatorname{rng}\left(I^{\prime}\right)$ properly extends $\mathrm{rng}(I)$ or there is $l<n$ such that $J^{\prime}(l)>J(l)$, hence $\rho\left(I^{\prime}, J^{\prime}\right)>\rho(I, J)$.

DEFINITION 4. The universe of $\mathfrak{C}(m, n, r)$ is the power set of $F(m, n, r)$ and the operators are

- the boolean operators,+- are union and set complement,

- the diagonal $\mathrm{d}_{x y}=\{f \in F(m, n, r): f(x, y)=I d\}$,

- the cylindrifier $\mathrm{c}_{x}(X)=\left\{f \in F(m, n, r): \exists g \in X f \equiv_{x} g\right\}$ and

- the polyadic $\mathbf{s}_{\tau}(X)=\{f \in F(m, n, r): f \tau \in X\}$, for $x, y<m, X \subseteq F(m, n, r)$ and $\tau: m \rightarrow m$.

Let $x, y<m$ and let $b \in \operatorname{Bin}(n, r)$. Define

$$
b_{x, y}=\{f \in F(m, n, r): f(x, y)=b\} \quad \in \mathfrak{C}(m, n, r)
$$

Observe, for any $x, y, z<m$ and $\lambda, \mu, \rho \in \operatorname{Bin}(n, r)$, that

$$
(u, v, w) \in \text { Forb } \Longleftrightarrow u_{x, y} \cap v_{y, z} \cap w_{x, z}=\emptyset,
$$

in particular we will use the case $(x, y, z)=(0,1,2)$, later.

LEMMA 5. For $3 \leq m, 2 \leq n$ and $r<\omega$ the algebra $\mathfrak{C}(m, n, r)$ satisfies all of the axioms defining QPEA $\mathbf{A}_{m}$ (see definition 15, noting that for finite $m, \quad \mathbf{P E A}_{m}$ is the same as $\mathbf{Q P E \mathbf { A } _ { m }}$ ) except, perhaps, the commutativity of cylindrifiers $\mathrm{c}_{x} \mathrm{c}_{y}(X)=\mathrm{c}_{y} \mathrm{c}_{x}(X)$. 
Proof. Routine.

LEMMA 6. If $3 \leq m \leq m^{\prime}$ then $\mathfrak{C}(m, n, r) \cong \mathfrak{N r}_{m} \mathfrak{C}\left(m^{\prime}, n, r\right)$. $X\}$.

Proof. The isomorphism maps $X \subseteq F(m, n, r)$ to $\left\{f \in F\left(m^{\prime}, n, r\right): f \uparrow_{m \times m} \in\right.$

LEMMA 7. For $3 \leq m \leq n$ and $r<\omega, \quad \mathfrak{C}(m, n, r) \in \mathbf{Q P E A}_{m}$.

Proof. If $r=0$ then $\operatorname{Bin}(n, r)=\{I d\}$ so $\mathfrak{C}(m, n, 0)$ is the trivial algebra hence $\mathfrak{C}(m, n, 0) \in \mathbf{Q P E A}_{m}$. Now assume $r>0$. In view of lemma 5 we only have to check the commutativity of cylindrifiers: $\mathrm{c}_{x} \mathrm{c}_{y} X=\mathrm{c}_{y} \mathrm{c}_{x} X$, for $x, y<m$. This equation is trivial if $x=y$ so assume not. By complete additivity, it suffices to check the case where $X$ is an atom, $X=\{f\}$ for some $f \in F(m, n, r)$, that is we must show that $g \in \mathrm{c}_{x} \mathrm{c}_{y}\{f\} \Longleftrightarrow g \in \mathrm{c}_{y} \mathrm{c}_{x}\{f\}$. Thus given $g \equiv_{x y} f$, it suffices to find $h \in F(m, n, r)$ such that $f \equiv_{x} h \equiv_{y} g$. If there is $z<m, z \neq x, y$ and $f(y, z)=I d$ then the required $h$ is $g[y / z]$, or if $g(z, x)=I d$ the required $h$ is $f[x / z]$. Suppose there is no such $z$, so for each $z<m, z \neq x, y$ we have $f(y, z), g(x, z) \in a$. Let $h: m \times m \rightarrow \operatorname{Bin}(n, r)$ be identical to $f$ on pairs not involving $x$, be identical to $g$ on pairs not involving $y$ (this is welldefined, since $\left.f \equiv_{x y} g\right)$ and let $h(x, y)=h(y, x)=a^{0}(i, 0)$, where $i$ is the least number below $n-1$ such that it is not the case that there is $z \neq x, y<m$ and $f(y, z), g(x, z) \in a(i,-)$. Since $m \leq n$ and there are only $m-2$ possible values of $z$ in $m \backslash\{x, y\}$ and $n-1$ possible values of $i$, such an $i$ must exist. This defines $h$. It is now easy to check that $h \in F(m, n, r)$.

We can now prove theorem 1 (I): if $3 \leq m \leq n$ and $r<\omega$ then $\mathfrak{C}(m, n, r) \cong$ $\mathfrak{N r}_{m}(\mathfrak{C}(n, n, r))$ by lemma 6 and $\mathfrak{C}(n, n, r) \in \mathbf{Q P E A}_{n}$ by lemma 7 , so $\mathfrak{C}(m, n, r) \in$ $\mathrm{Nr}_{m} \mathbf{Q P E A}_{n}$. Next, we prove theorem 1 (II).

LEMMA 8. Let $3 \leq m<\omega, 2 \leq n<\omega, r<\omega . \mathfrak{R d}_{\mathbf{S c}} \mathfrak{C}(m, n, r) \notin$ $S \mathrm{Nr}_{m} \mathbf{S c}_{n+1}$.

Proof. Suppose, for contradiction, that $\mathcal{X} \in \mathbf{S c}_{n+1}$ and $/: \mathfrak{R} \mathfrak{d}_{\mathbf{S c}} \mathfrak{C}(m, n, r) \rightarrow$ $\mathfrak{N r}_{m} \mathcal{X}$ is an isomorphism. Let $B \subseteq \operatorname{Bin}(n, r)$ and let $i<j<n+1$. Define

$$
\alpha(B, i, j)=\sum\left\{\mathrm{s}_{[0 / i]} \mathrm{s}_{[1 / j]} f^{\prime}: f \in F(m, n, r), f(0,1) \in B\right\} \quad \in \mathcal{X}
$$

For $b \in \operatorname{Bin}(n, r)$ we may write $\alpha(b, i, j)$ instead of $\alpha(\{b\}, i, j)$. By additivity of the substitutions

$$
\sum_{b \in \operatorname{Bin}(n, r)} \alpha(b, i, j)=1 .
$$

Further, for $i<j<k<n+1$ and $b, c, d \in \operatorname{Bin}(n, r)$, we have

$$
\begin{aligned}
& \alpha(b, i, j) \cdot \alpha(c, j, k) \cdot \alpha(d, i, k)=0 \\
& \Longleftrightarrow \Sigma_{f \in F(m, n, r), f(0,1)=b} \mathrm{~S}_{[0 / i]} \mathrm{S}_{[1 / j]} f^{\prime} \cdot \Sigma_{g(0,1)=c} \mathrm{~S}_{[0 / j]} \mathrm{S}_{[1 / k]} g^{\prime} \cdot \Sigma_{h(0,1)=d} \mathrm{~S}_{[0 / i]} \mathrm{S}_{[1 / k]} h^{\prime}=0 \\
& \Longleftrightarrow \neg \exists p \in F(m, n, r)(p(i, j)=b, p(j, k)=c, p(i, k)=d) \\
& \Longleftrightarrow(b, c, d)(๘) F o r b
\end{aligned}
$$


Let

$$
\beta^{k}=\alpha\left(a^{k}(0,0), 0, n\right) \cdot \prod_{i<n} \alpha(I d, 0, i) \in \mathcal{X} .
$$

Since there is $f \in F(m, n, r)$ with $f(0,1)=a^{k}(0,0)$, letting $\sigma: n+1 \rightarrow n+1$ be the function $\sigma(1)=n, \sigma(i)=0(i<n+1, i \neq 1)$, we have $0 \neq \mathrm{s}_{\sigma} f^{\prime} \leq \beta^{k}$, and clearly for $k \neq k^{\prime}<\psi(n, r)$ we have $\beta^{k} \cdot \beta^{k^{\prime}} \leq \alpha\left(a^{k}(0,0), 0, n\right) \cdot \alpha\left(a^{k^{\prime}}(0,0), 0, n\right)=$ 0 .

Let $S_{0}=\left\{\beta^{k}: k<\psi(n, r)\right\}$. We will prove by induction over $t$ that if $t \leq(n-1) r$ there is a set $S_{t}$ with $\left|S_{t}\right|>\kappa((n-1) r,(n-1) r-t)$ and functions $I_{t}:\{0, \ldots, n-1\} \rightarrow\{0, \ldots, n-2\}, J_{t}:\{0, \ldots, n-1\} \rightarrow\{0, \ldots, r-1\}$, such that for all $\beta, \beta^{\prime} \in S_{t}$

1. if $l<n$ then $\beta \leq \alpha\left(a\left(I_{t}(l), J_{t}(l)\right), l, n\right)$,

2. there is $k<\psi(n, r)$ unique to $\beta \in S_{t}$ such that $\beta \leq \alpha\left(a^{k}, 0, n\right)$,

3. $\mathrm{c}_{n} \beta=\mathrm{c}_{n} \beta^{\prime}$,

To see that the case $t=0$ holds: let $I_{0}(i)=0, J_{0}(i)=0($ all $i<n)$.

Given functions $I_{t}, J_{t}$ as above and $i<n-1$ let the index of $i$ with respect to $I_{t}, J_{t}$ be

$$
\operatorname{ind}\left(i, I_{t}, J_{t}\right)=\max \left(\left\{J_{t}(l): l<n-1, I_{t}(l)=i\right\} \cup\{-1\}\right) .
$$

Define the $\operatorname{rank} \rho\left(I_{t}, J_{t}\right)=\sum_{i<n-1} \operatorname{ind}\left(i, I_{t}, J_{t}\right)$. Observe that $\operatorname{ind}\left(0, I_{0}, J_{0}\right)=0$ and $\operatorname{ind}\left(i, I_{0}, J_{0}\right)=-1$ for $0<i<n-1$, so $\rho\left(I_{0}, J_{0}\right)=0+(n-2) \times(-1)=2-n$. We also assume, inductively,

4. $\rho\left(I_{t}, J_{t}\right) \geq 2-n+t$.

We have seen that this last inductive condition also holds for $t=0$.

Let $0 \leq t<(n-1) r$ and assume these properties hold. Since $\left|\operatorname{dom}\left(I_{t}\right)\right|=n$ and $\left|\operatorname{rng}\left(I_{t}\right)\right| \leq n-1$ there must be $u<v<n$ with $I_{t}(u)=I_{t}(v)$. Pick such a pair $(u, v)$ and let $l=u$ if $J_{t}(u) \leq J_{t}(v)$, else let $l=v$. Note, by choice of $l$, that if $I^{\prime}, J^{\prime}$ are functions identical to $I, J$, respectively, except perhaps on $l$, then $\rho\left(I^{\prime}, J^{\prime}\right) \geq \rho(I, J)$.

Since $t<(n-1) r$ we have $\left|S_{t}\right|>\kappa((n-1) r,(n-1) r-t) \geq \kappa((n-1) r, 1)=$ 1. Fix some $\beta_{0} \in S_{t}$. For each $\beta \in S_{t} \backslash\left\{\beta_{0}\right\}$, since $c_{n} \beta=c_{n} \beta_{0}$, we have $\mathrm{c}_{n} \mathrm{c}_{l} \mathrm{~s}_{[n / l]} \mathrm{c}_{l}(\beta)=\mathrm{c}_{n} \mathrm{c}_{l}\left(\beta_{0}\right)$, hence $\mathrm{c}_{l} \beta_{0} \cdot \mathrm{s}_{[n / l]} \mathrm{c}_{l} \beta \neq 0$. By (4), there is $b \in$ $\operatorname{Bin}(n, r)$ such that

$$
\delta=\mathrm{c}_{l} \beta_{0} \cdot \mathrm{s}_{[n / l]} \mathrm{c}_{l} \beta \cdot \alpha(b, l, n) \neq 0 .
$$

We know that $\beta_{0} \leq \alpha\left(a^{k_{0}}(0,0), 0, n\right), \beta \leq \alpha\left(a^{k}(0,0), 0, n\right)$ for some $k_{0} \neq k<$ $\psi(n, r)$, so $\delta \leq \alpha\left(a^{k_{0}}(0,0), 0, l\right) \cdot \alpha\left(a^{k}(0,0)(0, n)\right) \cdot \alpha(b, l, n)$. By $(5),\left(a^{k_{0}}(0,0), a^{k}(0,0), b\right) \notin$ Forb and we cannot have $b=I d$. Hence $b=a^{k^{\prime}}(i, j)$ for some $i<n-1, j<$ $r, k^{\prime}<\psi(n, r)$. For $i<n, j<r$ let

$$
S(i, j)=\left\{\mathrm{c}_{l} \beta_{0} \cdot \mathrm{s}_{[n / l]} \mathrm{c}_{l} \beta \cdot \alpha\left(a^{k}(i, j), l, n\right): k<\psi(n, r), \beta \in S_{t} \backslash\left\{\beta_{0}\right\}\right\} \backslash\{0\} .
$$

By cardinalities, there are fixed $i_{0}<n-1$ and $j_{0}<r$ such that $\left|S\left(i_{0}, j_{0}\right)\right| \geq$ $\frac{\left|S_{t}\right|-1}{(n-1) r}>\frac{\kappa((n-1) r,(n-1) r-t)-1}{(n-1) r}=\kappa((n-1) r,(n-1) r-(t+1))$. Let $S_{t+1}=S\left(i_{0}, j_{0}\right)$, let $I_{t+1}$ be identical to $I_{t}$ except that $l \mapsto i_{0}$ and let $J_{t+1}$ be identical to $J_{t}$ except that $l \mapsto j_{0}$. If $i_{0} \notin \operatorname{rng}\left(I_{t}\right)$ then $\operatorname{Ind}\left(i_{0}, I_{t+1}, J_{t+1}\right)=j_{0} \geq 0>-1=$ 
$\operatorname{Ind}\left(i_{0}, I_{t}, J_{t}\right)$, otherwise for any $p<n+1$ if $I_{t}(p)=i_{0}$ then $j_{0}>J_{t}(p)$, by (5) applied to $(p, l, n)$, so $j_{0}=\operatorname{Ind}\left(i_{0}, I_{t+1}, J_{t+1}\right)>\operatorname{Ind}\left(i_{0}, I_{t}, J_{t}\right)$. Either way, $\rho\left(I_{t+1}, J_{t+1}\right)>\rho\left(I_{t}, J_{t}\right)$. Hence $S_{t+1}, I_{t+1}, J_{t+1}$ satisfies induction hypothesis 3 . The other induction hypotheses are straightforward.

By induction, the properties hold for all $t \leq(n-1) r$. Letting $t=(n-1) r$, we have a set $S_{(n-1) r}$ of size strictly greater than $\kappa((n-1) r,(n-1) r-(n-1) r)=$ $\kappa((n-1) r, 0)=0$, i.e. non-empty, and there are functions $I_{t}, J_{t}$ of rank at least $(2-n)+((n-1) r)=(n-1)(r-1)+1$, an impossibility since for each $i<n-1$ the maximum index $i$ can have is $r-1$, hence the maximum possible rank is $(n-1)(r-1)$. We conclude that $\mathfrak{R} \mathfrak{d}_{\mathbf{S c}} \mathfrak{C}(m, n, r) \notin S \mathrm{Nr}_{m} \mathbf{S c}_{n+1}$.

We now concentrate on proving (III), that $\Pi_{r / U} \mathfrak{C}(m, n, r) \in S \mathrm{Nr}_{m} \mathbf{Q P E A}_{n+1}$, for any non-principal ultrafilter $U$. A standard ultraproduct argument shows that $\Pi_{r / U} \mathfrak{C}(m, n, r) \cong \mathfrak{C}\left(m, n, \Pi_{r / U} r\right)$ so we have to prove that $\mathfrak{C}(m, n, \rho) \in$ $S \mathrm{Nr}_{m} \mathbf{Q P E A}_{n+1}$, where $\rho=\Pi_{r / U} r$. Note that $\rho$ is a linear order containing an infinite ascending chain. First we define a game.

DEFINITION 9. Let $m, n<\omega$, let $\rho$ be a linear order and let $\Lambda=(n+1)^{3}$. An $m$-hypernetwork $h=(f, g)$ consists of some $f \in F(m, n, \rho)$ and a ternary function $g:{ }^{3} m \rightarrow \Lambda$ such that for all $x, y, z, x^{\prime}, y^{\prime}, z^{\prime}<m$, if $f\left(x, x^{\prime}\right)=$ $f\left(y, y^{\prime}\right)=f\left(z, z^{\prime}\right)=I d$ then $g(x, y, z)=g\left(x^{\prime}, y^{\prime}, z^{\prime}\right)$. For $X \subseteq m$ we say that $h=(f, g)$ is strict over $X$ if $x \neq y \in X \Rightarrow f(x, y) \neq I d$. If $m^{\prime} \leq m$ and $h=(f, g)$ is an m-hypernetwork then $h \uparrow_{m^{\prime}}$ denotes the $m^{\prime}$-hypernetwork obtained from $h$ by restriction to $m^{\prime}$. As before, for $x, y<m$ we write $(f, g) \equiv_{x y}\left(f^{\prime}, g^{\prime}\right)$ if for all $v, w, z \in m \backslash x, y$ we have $f(v, w)=f^{\prime}(v, w)$ and $g(v, w, z)=g^{\prime}(v, w, z)$, also we write $\equiv_{x}$ instead of $\equiv_{x x}$.

We define a game $G=G(m, n, \rho)$ as follows. A play of $G$ is a sequence $h_{0}, h_{1}, \ldots, h_{t}, \quad(t<\omega)$ of $(n+1)$-hypernetworks. In round $t<\omega$ of the game, $\forall$ plays either an $m$-dimensional move $\theta$ by choosing any m-hypernetwork $\theta$ or an amalgamation move $(u, v, \sigma, \tau, x, y)$ where $u, v<t, \sigma, \tau: n+1 \rightarrow n+1, x, y<$ $n+1$ and $h_{u} \sigma \equiv_{x y} h_{v} \tau$. In response to an $m$-dimensional move $\theta, \exists$ must play a $(n+1)$-hypernetwork $h_{t}$ such that $h_{t} \Upsilon_{m}=\theta$. In response to an amalgamation move $(u, v, \sigma, \tau, x, y), \exists$ must play a $(n+1)$-hypernetwork $h_{t}$ such that $h_{u} \sigma \equiv_{x} h_{t} \equiv_{y} h_{v} \tau$. If she fails to provide such a response to either kind of move then she loses the play in that round. If $\exists$ does not lose in any of the $\omega$ rounds of $G$ then she wins the play.

LEMMA 10. Let $3 \leq m<n<\omega$ and let $\rho$ be a linear order containing an infinite ascending sequence. $\exists$ has a winning strategy in $G(m, n, \rho)$.

Proof. Let $j_{0}<j_{1}<j_{2} \ldots \in \rho$ be an infinite ascending sequence, let $J=\left\{j_{0}, j_{1}, \ldots\right\} \subseteq \rho$. We describe $\exists$ 's strategy. Consider round $t$ of a play of the game. Suppose, inductively, that $\exists$ has successfully implemented her strategy in all previous rounds $s<t$, the play so far is $h_{0}, h_{1}, \ldots h_{t-1}$. Suppose $\forall$ plays an $m$-dimensional move $\theta$. Let $\sigma:(n+1) \rightarrow m$ be the function defined by

$$
\sigma(i)= \begin{cases}i & (i<m) \\ 0 & (m \leq i<n+1)\end{cases}
$$

$\exists$ plays the hypernetwork $\theta \sigma$. Observe that if $X \subseteq(n+1)$ and $|X|>m$ then $\theta \sigma$ is not strict over $X$. 
Now suppose $\forall$ plays an amalgamation move $(u, v, \sigma, \tau, x, y)$ where $h_{u} \sigma \equiv_{x y}$ $h_{v} \tau$. To avoid trivialities assume $x \neq y$. $\exists$ is required to find $h_{t}=\left(f_{t}, g_{t}\right)$ such that $h_{u} \sigma \equiv_{x} h_{t} \equiv_{y} h_{v} \tau$. These equivalences uniquely determine the value of $f_{t}$ on any pair from $n+1$ except $(x, y)$ and $(y, x)$ and they determine the value of $g_{t}$ on any triple from $n+1$ except those involving both $x$ and $y$. If there is $w<t$ and $\rho: n+1 \rightarrow n+1$ such that $h_{u} \sigma \equiv_{x} h_{w} \rho \equiv_{y} h_{v} \tau$ then $\exists$ lets $h_{t}=h_{w} \rho$ (if there is more than one possible solution, then any will do). Since such a move by $\forall$ is clearly superfluous we will assume henceforth that $\forall$ never makes such a move. Furthermore, we will assume that if $\forall$ plays the amalgamation move $(u, v, \sigma, \tau, x, y)$ then there is no $u^{\prime}<u$ and $\sigma^{\prime}: n+1 \rightarrow n+1$ such that $h_{u} \sigma \equiv_{x} h_{u^{\prime}} \sigma^{\prime}$ (if such a $u^{\prime}<u$ and $\sigma^{\prime}$ existed then $\forall$ could instead play $\left.\left(u^{\prime}, v, \sigma^{\prime}, \tau, x, y\right)\right)$ and there is no $v^{\prime}<v$ and $\tau^{\prime}: n+1 \rightarrow n+1$ such that $h_{v} \tau \equiv{ }_{y} h_{v^{\prime}} \tau^{\prime}$.

Now, although we have not yet entirely defined $f_{t}$, for each $x^{\prime}, y^{\prime}<n+1$ we already know whether $f_{t}\left(x^{\prime}, y^{\prime}\right)=I d$ or not (we cannot have $f_{t}(x, y)=I d$, by our assumption about $\forall$-moves). For any $x_{1}, y_{1}, z_{1}, x_{2}, y_{2}, z_{2}<n+1$ we write $\left(x_{1}, y_{1}, z_{1}\right) \sim\left(x_{2}, y_{2}, z_{2}\right)$ iff $f_{t}\left(x_{1}, x_{2}\right)=f_{t}\left(y_{1}, y_{2}\right)=f_{t}\left(z_{1}, z_{2}\right)=I d$.

First $\exists$ defines $g_{t}:^{3}(n+1) \rightarrow \Lambda$ by defining $g_{t}$ on all triples involving both $x$ and $y$ in such a way that if $\bar{x}$ is any triple involving $x$ and $y$ and if $\bar{y}$ is any triple of elements of $(n+1)$ then $g_{t}(\bar{x})=g_{t}(\bar{y}) \Longleftrightarrow(\bar{x} \sim \bar{y})$. Since $\sim$ is clearly an equivalence relation and since $\Lambda=(n+1)^{3}$, the range of $g_{t}$ is large enough to allow this.

Secondly, $\exists$ defines $f_{t} \in F(n+1, n, \rho)$ by letting $f_{t}(x, y)=a^{0}(i, j)$ where

- $j \in J$ is greater than each element of the finite set $\left\{j^{\prime} \in J: \exists s<t, x^{\prime}, y^{\prime}<\right.$ $\left.n+1 f_{u}\left(x^{\prime}, y^{\prime}\right) \in a\left(-, j^{\prime}\right)\right\}$, least possible subject to that.

- $i<n-1$ is least such that there is no $w<n+1$ and $j \in \rho \backslash J$ with $f_{s} \sigma(y, w), f_{v} \tau(w, x) \in a(i, j)$.

We will prove that the strategy may be implemented, in particular the $i<n-1$ required in the second part may always be found. To prove our claim, suppose for contradiction that there are $w_{0}, w_{1}, \ldots, w_{n-2}<n+1$ such that for each $i<n-1$ there is $j \in \rho \backslash J$ and $f_{u} \sigma\left(y, w_{i}\right),\left(f_{v} \tau\right)\left(w_{i}, x\right) \in a(i, j)$. Observe that $f_{u}$ is strict over $\left\{\sigma(y), \sigma\left(w_{i}\right): i<n-1\right\}$, so $f_{u}$ was itself played in response to an amalgamation move, say $\left(u^{\prime}, v^{\prime}, \sigma^{\prime}, \tau^{\prime}, x^{\prime}, y^{\prime}\right)$. By our assumption that there is no $u^{*}<u$ and $\sigma^{*}$ such that $f_{u} \sigma \equiv_{x} f_{u^{*}} \sigma^{*}$, we see that $\left\{\sigma\left(y^{\prime}\right), \sigma\left(x^{\prime}\right)\right\} \subseteq$ $\left\{y, w_{0}, w_{1}, \ldots, w_{n-2}\right\}$. Inductively, $\exists$ chose $f_{u}\left(\sigma\left(y^{\prime}\right), \sigma\left(x^{\prime}\right)\right) \in a\left(-, k^{\prime}\right)$ for some $k^{\prime} \in K$, hence $\left\{\sigma\left(y^{\prime}\right), \sigma\left(x^{\prime}\right)\right\} \subseteq\left\{w_{0}, \ldots, w_{n-2}\right\}$. Similarly, $f_{v}$ was played in response to an amalgamation move $\left(u^{*}, v^{*}, \sigma^{*}, \tau^{*}, x^{*}, y^{*}\right), f_{v}\left(\tau\left(y^{*}\right), \tau\left(x^{*}\right)\right) \in$ $a\left(-, k^{*}\right)$ (some $k^{*} \in K$ ) and $\left\{\tau\left(y^{*}\right), \tau\left(x^{*}\right)\right\} \subseteq\left\{w_{0}, \ldots, w_{n-2}\right\}$. By uniqueness of $k^{\prime}$ and $k^{*}$ we deduce that $k^{\prime}=k^{*}, u=v$ and $\left\{\sigma\left(y^{\prime}\right), \sigma\left(x^{\prime}\right)\right\}=\left\{\tau\left(y^{*}\right), \tau\left(x^{*}\right)\right\}$. When $\exists$ played $f_{u}$ she ensured that for each $w_{h}(h<n-1)$ the label $g_{u}\left(\sigma\left(y^{\prime}\right), \sigma\left(x^{\prime}\right), \sigma\left(w_{h}\right)\right)$ is unique but it is equal to $g_{v}\left(\tau\left(y^{\prime}\right), \tau\left(x^{\prime}\right), \tau\left(w_{h}\right)\right.$ ) (since $g_{u} \sigma \equiv_{x y} g_{v} \tau$ ), hence $\sigma\left(w_{h}\right)=\tau\left(w_{h}\right)$. But then, define $\rho:(n+1) \rightarrow(n+1)$ by $\rho(v)=\sigma(v)$, for $v \in(n+1) \backslash\{x\}$, and $\rho(x)=\tau(x)$. Then $h_{u} \sigma \equiv_{x} h_{u} \rho \equiv_{y} h_{v} \sigma$, contrary to our assumption. This proves the claim and proves that $\exists$ 's strategy can always be implemented. 
By choice of $i, j$ it is clear that $f_{t}$ avoids all forbidden triples so indeed $f_{t} \in$ $F(n+1, n, \rho)$.

LEMMA 11. Let $3 \leq m<n<\omega$ and let $\rho$ be a countable linear order containing an infinite ascending sequence. Then $\mathfrak{C}(m, n, \rho) \in \mathrm{Nr}_{m} \mathbf{Q P E A}_{n+1}$.

Proof. Consider a play of $G(m, n, \rho)$ in which $\exists$ plays her winning strategy and $\forall$ plays all possible $m$-dimensional moves and all possible amalgamation moves. Since $\rho$ is countable, this can be scheduled. Let $H$ be the set of all hypernetworks occurring in the play. As in definition 4, the power set $\wp(H)$ is the universe of a QPEA $\mathbf{A}_{n+1}$-type algebra $\mathfrak{C}$, where $\mathrm{d}_{i j}=\{(f, g) \in H: f(i, j)=$ $I d\}, \mathrm{c}_{i}(X)=\left\{h \in H: \exists h^{\prime} \in X, h^{\prime} \equiv_{i} h\right\}$ and $s_{\tau}(X)=\{h \in H: h \tau \in X\}$, for $i, j<n+1, \tau:(n+1) \rightarrow(n+1)$. As with lemma 5 it is easy to see that $\mathfrak{C}$ satisfies all the $\mathbf{Q P E A} \mathbf{A}_{n+1}$ axioms other than commutativity of cylindrifiers, and since $H$ is closed under amalgamation, commutativity holds too, so $\mathfrak{C} \in \mathbf{Q P E A}_{n+1}$. The map $\lambda: \mathfrak{C}(m, n, \rho) \rightarrow \mathfrak{N r}_{m} \mathfrak{C}$ defined by $\iota(f)=\left\{\left(f^{\prime}, g^{\prime}\right) \in H: f^{\prime} \uparrow_{m}=f\right\}$ is easily shown to be an isomorphism.

Since $\Pi_{r / U} \mathfrak{C}(m, n, r) \cong \mathfrak{C}\left(m, n, \Pi_{r / U} r\right)$ and $\Pi_{r / U} r$ contains an infinite ascending sequence, this proves theorem 1 (III) and completes the proof of theorem 1 .

Infinite dimensional case. Now we prove the infinite dimensional case, by lifting the dimensions for the finite case to the transfinite; a trick due to Monk; witness [5, theorem 3.2.87] where Monk lifts his classical non finite axiomatizability result for $\mathbf{R C A}_{n}(n>2)$ to the transfinite. Our proof has the same structure as the finite dimensional case, but naturally we need an infinite dimensional quasi- polyadic equality algebra. Let $n$ be an infinite ordinal. For each finite subset $\Gamma \subseteq n$ let

$$
\rho_{\Gamma} \text { be the unique order preserving bijection from }|\Gamma| \text { onto } \Gamma \text {. }
$$

Let $I=\{\Gamma: \Gamma \subseteq n,|\Gamma|<\omega\}$. For each $\Gamma \in I$, let $M_{\Gamma}=\{\Delta \in I: \Gamma \subseteq \Delta\}$, and let $F$ be an ultrafilter on $I$ such that $\forall \Gamma \in I, M_{\Gamma} \in F$ (such an ultrafilter exists because $M_{\Gamma_{1}} \cap M_{\Gamma_{2}}=M_{\Gamma_{1} \cup \Gamma_{2}}$ ). Let $r<\omega, 1 \leq k<\omega, \Gamma \in I$, and let $\mathfrak{C}_{\Gamma}^{r}$ be an algebra similar to $\mathbf{Q P E A} \mathbf{A}_{n}$ such that

$$
\mathfrak{R d}^{\rho_{\Gamma}} \mathfrak{C}_{\Gamma}^{r}=\mathfrak{C}(|\Gamma|,|\Gamma|+k, r) .
$$

Let

$$
\mathfrak{B}^{r}=\prod_{\Gamma / F} \mathfrak{C}_{\Gamma}^{r} .
$$

THEOREM 12. Let $U$ be any non-principal ultraproduct over $\omega$.

1. $\mathfrak{B}^{r} \in S \mathrm{Nr}_{n} \mathbf{Q P E A}_{n+k}$,

2. $\mathfrak{R d} \mathbf{S c} \mathfrak{B}^{r} \notin S \mathrm{Nr}_{n} \mathbf{S c}_{n+k+1}$ and

3. $\Pi_{r / U} \mathfrak{B}^{r} \in S \mathrm{Nr}_{n} \mathbf{Q P E A} \mathbf{A}_{n+k+1}$.

But first a lemma.

LEMMA 13. Let $n$ be an infinite ordinal, let $X$ be any finite subset of $n$, let $I=\{\Gamma: X \subseteq \Gamma \subseteq n,|\Gamma|<\omega\}$. For each $\Gamma \in I$ let $M_{\Gamma}=\{\Delta \in I: \Delta \supseteq \Gamma\}$ and let $F$ be any ultrafilter over $I$ such that for all $\Gamma \in I$ we have $M_{\Gamma} \in F$. Let 
$\mathcal{A}_{\Gamma}, \mathcal{B}_{\Gamma}$ be $\mathbf{Q P E A}{ }_{n}$-type algebras. If for each $\Gamma \in I$ we have $\mathfrak{R d}^{\rho_{\Gamma}} \mathcal{A}_{\Gamma}=\mathfrak{R d}^{\rho_{\Gamma}} \mathcal{B}_{\Gamma}$ then $\Pi_{\Gamma / F} \mathcal{A}_{\Gamma}=\Pi_{\Gamma / F} \mathcal{B}_{\Gamma}$.

Furthermore, if $\mathfrak{R d}^{\rho_{\Gamma}} \mathcal{A}_{\Gamma} \in \mathbf{Q P E A} \mathbf{A}_{|\Gamma|}$ for each $\Gamma \in I$, then $\Pi_{\Gamma / F} \mathcal{A}_{\Gamma} \in \mathbf{Q P E A} \mathbf{A}_{n}$.

Proof. Standard proof, by Łoś' theorem. For the first part, note that the universe of $\Pi_{\Gamma / F} \mathcal{A}_{\Gamma}$ is identical to that of $\Pi_{\Gamma / F} \mathfrak{R d}^{\rho_{\Gamma}} \mathcal{A}_{\Gamma}$ which is identical to the universe of $\Pi_{\Gamma / F} \mathcal{B}_{\Gamma}$, by the assumption in the first part of the lemma. Each operator $o$ of $\mathbf{Q P E A}_{n}$ is the same for both ultraproducts because $\{\Gamma \in I$ : $\left.\operatorname{dim}(o) \subseteq \operatorname{rng}\left(\rho_{\Gamma}\right)=\Gamma\right\} \in F$.

For the second part, it suffices to prove that each of the defining axioms for QPEA $_{n}$ holds for $\Pi_{\Gamma / F} \mathcal{A}_{\Gamma}$. Let $\sigma=\tau$ be one of the defining equations for $\mathbf{Q P E A}_{n}$, the number of dimension variables involved is certainly finite, indeed it can be at most four (see definition 15.8). Take any $i, j, k, l \in n$, we must prove that $\Pi_{\Gamma / F} \mathcal{A}_{\Gamma} \models \sigma(i, j, k, l)=\tau(i, j, k, l)$. If $i, j, k, l \in \operatorname{rng}\left(\rho_{\Gamma}\right)$, say $i=$ $\rho_{\Gamma}\left(i_{0}\right), j=\rho_{\Gamma}\left(j_{0}\right), k=\rho_{\Gamma}\left(r_{0}\right), l=\rho_{\Gamma}\left(l_{0}\right)$, then $\mathfrak{R d}^{\rho_{\Gamma}} \mathcal{A}_{\Gamma} \models \sigma\left(i_{0}, j_{0}, k_{0}, l_{0}\right)=$ $\tau\left(i_{0}, j_{0}, k_{0}, l_{0}\right)$, since $\mathfrak{R o}^{\rho_{\Gamma}} \mathcal{A}_{\Gamma} \in \mathbf{Q P E A}_{|\Gamma|}$, so $\mathcal{A}_{\Gamma} \models \sigma(i, j, k, l)=\tau(i, j, k, l)$. Hence $\left\{\Gamma \in I: \mathcal{A}_{\Gamma} \mid=\sigma(i, j, k, l)=\tau(i, j, k, l)\right\} \supseteq\left\{\Gamma \in I: i, j, k, l \in \operatorname{rng}\left(\rho_{\Gamma}\right\} \in F\right.$, hence $\Pi_{\Gamma / F} \mathcal{A}_{\Gamma}=\sigma(i, j, k, l)=\tau(i, j, k, l)$. Thus $\Pi_{\Gamma / F} \mathcal{A}_{\Gamma} \in \mathbf{Q P E A} \mathbf{A}_{n}$.

Proof of TheOREM 12. For the first part, for each $\Gamma \in I$ we know that $\mathfrak{C}(|\Gamma|+k,|\Gamma|+k, r) \in \mathbf{Q P E A}_{|\Gamma|+k}$ and $\mathfrak{N \mathfrak { r }}|\Gamma| \mathfrak{C}(|\Gamma|+k,|\Gamma|+k, r) \cong \mathfrak{C}(|\Gamma|,|\Gamma|+k, r)$ (by lemmas 6 and 7 ). Let $\sigma_{\Gamma}$ be the one to one function $(|\Gamma|+k) \rightarrow(n+k)$ where $\rho_{\Gamma} \subseteq \sigma_{\Gamma}$ and $\sigma_{\Gamma}(|\Gamma|+i)=n+i$ for each $i<k$. Let $\mathcal{A}_{\Gamma}$ be an algebra similar to a $\mathbf{Q P E A} \mathbf{A}_{n+k}$ such that $\mathfrak{R o}{ }^{\sigma_{\Gamma}} \mathcal{A}_{\Gamma}=\mathfrak{C}(|\Gamma|+k,|\Gamma|+k, r)$. By the second part of lemma 13, with $n+k$ in place of $n, m \cup\{n+i: i<k\}$ in place of $X$, $\{\Gamma \subseteq n+k:|\Gamma|<\omega, X \subseteq \Gamma\}$ in place of $I$, and with $\sigma_{\Gamma}$ in place of $\rho_{\Gamma}$, we know that $\Pi_{\Gamma / F} \mathfrak{A}_{\Gamma} \in \mathbf{Q P E A} \mathbf{A}_{n+k}$.

We prove that $\mathfrak{B}^{r} \subseteq \mathfrak{N r}_{n} \Pi_{\Gamma / F} \mathcal{A}_{\Gamma}$. Recall that $\mathfrak{B}^{r}=\Pi_{\Gamma / F} \mathfrak{C}_{\Gamma}^{r}$. For each $\Gamma \in I$,

$$
\begin{aligned}
\mathfrak{R d}^{\rho_{\Gamma}} \mathfrak{C}_{\Gamma}^{r} & =\mathfrak{C}((|\Gamma|,|\Gamma|+k, r) \\
& \cong \mathfrak{N r}_{|\Gamma|} \mathfrak{C}(|\Gamma|+k,|\Gamma|+k, r) \\
& =\mathfrak{N r}_{|\Gamma|} \mathfrak{R d}^{\sigma} \mathfrak{A}_{\Gamma} \\
& =\mathfrak{R d}^{\sigma} \mathfrak{N \mathfrak { r }}_{\Gamma} \mathfrak{A}_{\Gamma} \\
& =\mathfrak{R d}^{\rho_{\Gamma}} \mathfrak{N r}_{\Gamma} \mathfrak{A}_{\Gamma} .
\end{aligned}
$$

By the first part of lemma 13 we deduce that $\Pi_{\Gamma / F} \mathfrak{C}_{\Gamma}^{r} \cong \Pi_{\Gamma / F} \mathfrak{N r}_{\Gamma} \mathfrak{A}_{\Gamma} \subseteq \mathfrak{N r}_{n} \Pi_{\Gamma / F} \mathfrak{A}_{\Gamma}$, proving (1).

Now we prove (2), $\mathfrak{R} \mathfrak{d}_{\mathbf{S c}} \mathfrak{B}^{r} \notin S \mathrm{Nr}_{n} \mathbf{S c}_{n+k+1}$. For this assume, seeking a contradiction, that $\mathfrak{R} \mathfrak{d}_{\mathbf{S c}} \mathfrak{B}^{r} \in S \mathrm{Nr}_{n} \mathbf{S c}_{n+k+1}$, i.e. $\mathfrak{R d}_{\mathbf{S c}} \mathfrak{B}^{r} \subseteq \mathfrak{N r}_{n} \mathfrak{C}$, where $\mathfrak{C} \in$ $\mathbf{S c}_{n+k+1}$. Pick any $3 \leq m<\omega$ (e.g. take $m=3$ ) and let $\lambda: m+k+1 \rightarrow$ $n+k+1$ be the function defined by $\lambda(i)=i$ for $i<m$ and $\lambda(m+i)=n+i$ for $i<k+1$. Then $\mathfrak{R d}{ }^{\lambda}(\mathfrak{C}) \in \mathbf{S c}_{m+k+1}$ and $\mathfrak{R d} \mathfrak{d}_{m} \mathfrak{R d} \mathfrak{S c}_{\mathbf{S c}} \mathfrak{B}^{r} \subseteq \mathfrak{N r}_{m} \mathfrak{R d}{ }^{\lambda}(\mathfrak{C})$. Let $\mathfrak{A}=\mathfrak{R d}_{m} \mathfrak{R} \mathfrak{d}_{\mathbf{S c}} \mathfrak{B}^{r}$. We have just shown that

$$
\mathfrak{A} \in S \mathrm{Nr}_{m} \mathbf{S c}_{m+k+1} .
$$

For finite $m^{+}>m$, let

$$
x_{m^{+}}=\left\{f \in F\left(m^{+}, m^{+}+k, r\right): m \leq j<m^{+} \rightarrow \exists i<m f(i, j)=I d\right\} .
$$


Then $x_{m^{+}} \in \mathfrak{C}\left(m^{+}, m^{+}+k, r\right)$ and $\mathrm{c}_{i} x_{m^{+}} \cdot \mathrm{c}_{j} x_{m^{+}}=\mathrm{s}_{[i / j]} x_{m^{+}} \cdot \mathrm{s}_{[j / i]} x_{m^{+}}=x_{m^{+}}$ for distinct $i, j<m$. Furthermore

$$
I_{m^{+}}: \mathfrak{C}(m, m+k, r) \cong \mathfrak{R l}_{x_{m}+} \mathfrak{R} \mathfrak{d}_{m} \mathfrak{C}\left(m^{+}, m^{+}+k, r\right)
$$

via

$I_{n}(S)=\left\{f \in F\left(m^{+}, m^{+}+k, r\right): f\left\lceil m \times m \in S, \forall j\left(m \leq j<m^{+} \rightarrow \exists i<m f(i, j)=I d\right)\right\}\right.$.

So for each $\Gamma \in I, I_{|\Gamma|}$ is an isomorphism

$$
\mathfrak{C}(m, m+k, r) \cong \mathfrak{R I}_{x_{|\Gamma|}} \mathfrak{R} \mathfrak{d}_{m} \mathfrak{C}(|\Gamma|,|\Gamma|+k, r) .
$$

Let $x=\left(x_{|\Gamma|}: \Gamma \in I\right) / F\left(\in \mathfrak{B}^{r}\right)$ and let $\iota(b)=\left(I_{|\Gamma|} b: \Gamma \in I\right) / F$ for $b \in \mathfrak{C}(m, m+k, r)$. Then $\iota$ is an isomorphism from $\mathfrak{R} \mathfrak{d}_{\mathbf{S c}} C(m, m+k, r)$ into $\mathfrak{R} \mathfrak{d}_{\mathbf{S c}} \mathfrak{R l}_{x} \mathfrak{R} \mathfrak{d}_{m} \mathfrak{B}^{r}=\mathfrak{R l}_{x} \mathfrak{R} \mathfrak{d}_{m} \mathfrak{R} \mathfrak{d}_{\mathbf{S c}} \mathfrak{B}^{r}=\mathfrak{R i} \mathfrak{A l}_{x}$. Now $\mathfrak{A} \in S \mathrm{Nr}_{m} \mathbf{S c}_{m+k+1}$, by (6), and $\mathfrak{A}=\mathrm{s}_{[i / j]} x \cdot \mathbf{s}_{[j / i]} x=x$ for any distinct $i, j<m$ by Loś' theorem. It follows, by [4, theorem 2.6.38], that

$$
\mathfrak{R l}_{x} \mathfrak{A} \in S \mathrm{Nr}_{m} \mathbf{S c}_{m+k+1} .
$$

(Note that proof of the cited theorem makes no use of diagonal elements.) But then $\mathfrak{R} \mathfrak{d}_{\mathbf{S c}} \mathfrak{C}(m, m+k, r) \subseteq \mathfrak{R l}_{x} \mathfrak{A} \in S \mathrm{Nr}_{m} \mathbf{S c}_{m+k+1}$, contrary to theorem 1(II). This proves (2).

Now we prove theorem 12(3), putting the superscript $r$ to use. Recall that $\mathfrak{B}^{r}=\Pi_{\Gamma / F} \mathfrak{C}_{\Gamma}^{r}$, where $\mathfrak{C}_{\Gamma}^{r}$ has the type of QPEA $\mathbf{A}_{n}$ and $\mathfrak{R O}^{\rho_{\Gamma}} \mathfrak{C}_{\Gamma}^{r}=\mathfrak{C}(|\Gamma|,|\Gamma|+k, r)$. We know that $\Pi_{r / U} \mathfrak{R d}^{\rho_{\Gamma}} \mathfrak{C}_{\Gamma}^{r}=\Pi_{r / U} \mathfrak{C}(|\Gamma|,|\Gamma|+k, r) \subseteq \mathfrak{N i}_{|\Gamma|} \mathcal{A}_{\Gamma}$, for some $\mathcal{A}_{\Gamma} \in$ $\operatorname{QPEA}_{|\Gamma|+k+1}$.

Let $\lambda_{\Gamma}:|\Gamma|+k+1 \rightarrow n+k+1$ extend $\rho_{\Gamma}:|\Gamma| \rightarrow \Gamma(\subseteq n)$ and satisfy

$$
\lambda_{\Gamma}(|\Gamma|+i)=n+i
$$

for $i<k+1$. Let $\mathfrak{F}_{\Gamma}$ be a QPEA $\mathbf{A}_{n+k+1}$ type algebra such that $\mathfrak{R} \mathfrak{d}^{\lambda_{\Gamma}} \mathfrak{F}_{\Gamma}=\mathcal{A}_{\Gamma}$. As before, by the second part of lemma $13, \Pi_{\Gamma / F} \mathfrak{F}_{\Gamma} \in \mathbf{Q P E} \mathbf{A}_{n+k+1}$. And

$$
\begin{aligned}
\Pi_{r / U} \mathfrak{B}^{r} & =\Pi_{r / U} \Pi_{\Gamma / F} \mathfrak{C}_{\Gamma}^{r} \\
& \cong \Pi_{\Gamma / F} \Pi_{r / U} \mathfrak{C}_{\Gamma}^{r} \\
& \subseteq \Pi_{\Gamma / F} \mathfrak{N r}_{|\Gamma|} \mathcal{A}_{\Gamma} \\
& =\Pi_{\Gamma / F} \mathfrak{N \mathfrak { r }}_{|\Gamma|} \mathfrak{R \mathfrak { d } ^ { \lambda }}{ }^{\lambda_{\Gamma}} \mathfrak{F}_{\Gamma} \\
& \subseteq \mathfrak{N r}_{n} \Pi_{\Gamma / F} \mathfrak{F}_{\Gamma},
\end{aligned}
$$

proving the last part of the theorem.

COROLLARY 14. Let $n$ be an infinite ordinal, let $k \in \omega$. Let $\mathbf{K}$ be any class between $\mathbf{S c}$ and QPEA. Then $S \mathrm{Nr}_{n} \mathbf{K}_{n+k+1} \subset S \mathrm{Nr}_{n} \mathbf{K}_{n+k}$. Furthermore, $S \mathrm{Nr}_{n} \mathbf{K}_{n+k+1}$ is not finite schema axiomatisable over $S \mathrm{Nr}_{n} \mathbf{K}_{n+k}$.

The first part of the corollary is credited to Pigozzi in [4, page 464], for cylindric algebras; however it seems that Pigozzi did not publish his proof, and we have not found a published proof elsewhere. See [5, definition 4.1.4] for the precise definition of finitely schema axiomatisability and see [5, theorem 4.1.7] to see how non finite schema axiomatisability follows from theorem 12 .

We summarize the situation in figure 2. The first table addresses the case when $3 \leq n<\omega$ and the second table addresses the case when $n \geq \omega$. For 


\begin{tabular}{|c|ll|}
\hline Algebras & $\begin{array}{l}\text { Status of the Neat Embedding Problem } \\
\text { for } 3 \leq n<\omega, k<\omega\end{array}$ & Citation \\
\hline $\mathbf{D f}_{n}$ & $S \mathrm{Nr}_{n} \mathbf{D f}_{n+k}=\mathbf{D f} \mathbf{f}_{n}$ & {$[5$, therorem 5.1.31] } \\
$\mathbf{S c}_{n}$ & $S \mathrm{Nr}_{n} \mathbf{S c}_{n+k+1}$ is n.f.a. over $S \mathrm{Nr}_{n} \mathbf{S c}_{n+k}$ & Corollary 2 \\
$\mathbf{C A}_{n}$ & $S \mathrm{Nr}_{n} \mathbf{C A}_{n+k+1}$ is n.f.a. over $S \mathrm{Nr}_{n} \mathbf{C A}_{n+k}$ & {$[8$, theorem 15.1(4)] } \\
$\mathbf{Q P A}_{n}$ & $S \mathrm{Nr}_{n} \mathbf{Q P A}_{n+k+1}$ is n.f.a. over $S \mathrm{Nr}_{n} \mathbf{Q P A} \mathbf{A}_{n+k}$ & Corollary 2 \\
$\mathbf{Q P E A}_{n}$ & $S \mathrm{Nr}_{n} \mathbf{Q P E A}_{n+k+1}$ is n.f.a. over $S \mathrm{Nr}_{n} \mathbf{Q P E E A} \mathbf{A}_{n+k}$ & Corollary 2 \\
\hline
\end{tabular}

\begin{tabular}{|c|c|c|}
\hline Algebras & $\begin{array}{l}\text { Status of the Neat Embedding Problem for } n \geq \\
\omega, k<\omega\end{array}$ & Cit: \\
\hline $\mathrm{Df}_{i}$ & $S \mathrm{Nr}_{n} \mathbf{D \mathbf { f } _ { n + k }}=\mathbf{\mathbf { D f }} \mathbf{f}_{n}$ & {$[5$, th } \\
\hline Sc & . & \\
\hline $\mathbf{C A}_{n}$ & 1 is n.f.a. & \\
\hline $\mathbf{Q P A}_{n}$ & $S \mathrm{Nr}_{n}$ & \\
\hline QPEA $_{n}$ & $S \mathrm{Nr}_{n} \mathbf{Q P E A}_{n+k+1}$ is & Co \\
\hline & $S \mathrm{Nr}_{n}$ & \\
\hline & & \\
\hline
\end{tabular}

Figure 2. Summary of Neat Embedding Problem

$n=0,1$, the problem is trivial $\left(S \mathrm{Nr}_{n} \mathbf{K}_{n+k}=\mathbf{K}_{n}\right)$. For $n=2$, we have for $\mathbf{K} \in\{\mathbf{D f}, \mathbf{S C}, \mathbf{Q A}\}$ and $k>0, S \mathrm{Nr}_{2} \mathbf{K}_{2+k}=\mathbf{K}_{2}$. The $\mathbf{D f}$ case is trivial, the SC and QA cases follow from [5, theorem 5.4.33] without much ado. On the other hand, for $\mathbf{K} \in\{\mathbf{C A}, \mathbf{P E A}\}$ (where diagonal elements are present) and $m>0, \mathbf{S N i} \mathbf{K}_{2+k}=\mathbf{R} \mathbf{K}_{2}$ with $\mathbf{R} \mathbf{K}_{2}$ denoting representable algebras in $\mathbf{K}_{2}$. This follows from [5, theorems 3.2.65, 5.4.34].

\section{Appendix.}

DEFINITION 15. Substitution Algebra, Sc: [13].

Let $n$ be an ordinal. By a substitution algebra of dimension n, briefly an $\mathbf{S c}_{n}$, we mean an algebra

$$
\mathfrak{A}=\left(A,+,-, \mathrm{c}_{i}, \mathrm{~s}_{[i / j]}: i, j<n\right)
$$

where $(A,+,-)$ is a boolean algebra, $\mathbf{c}_{i}, \mathbf{s}_{[i / j]}$ are unary operations on $\mathfrak{A}$ (for $i, j<n)$ satisfying the following equations for all $i, j, k, l<n$ :

1. $\mathrm{c}_{i} 0=0, x \leq \mathrm{c}_{i} x, \mathrm{c}_{i}\left(x \cdot \mathrm{c}_{i} y\right)=\mathrm{c}_{i} x \cdot \mathrm{c}_{i} y$, and $\mathrm{c}_{i} \mathrm{c}_{j} x=\mathrm{c}_{j} \mathrm{c}_{i} x$,

2. $\mathrm{s}_{[i / i]} x=x$,

3. $\mathrm{s}_{[i / j]}$ is a boolean endomorphisms,

4. $\mathrm{s}_{[i / j]} \mathrm{c}_{i} x=\mathrm{c}_{i} x$,

5. $c_{i} \mathrm{~s}_{[i / j]} x=\mathrm{s}_{[i / j]} x$ whenever $i \neq j$,

6. $\mathrm{s}_{[i / j]} c_{k} x=c_{k} \mathrm{~s}_{[i / j]} x$, whenever $k \notin\{i, j\}$,

7. $\mathrm{c}_{i} \mathrm{~s}_{[j / i]} x=\mathrm{c}_{j} \mathrm{~s}_{[i / j]} x$,

8. $\mathrm{s}_{[j / i]} \mathrm{s}_{[l / k]} x=\mathrm{s}_{[l / k]} \mathrm{s}_{[j / i]} x$, whenever $|\{i, j, k, l\}|=4$,

9. $\mathrm{s}_{[i / j]} \mathrm{s}_{[i / k]} x=\mathrm{s}_{[i / k]} x$ if $i \neq k$,

10. $\mathrm{s}_{[l / i]} \mathrm{s}_{[j / l]} x=\mathrm{s}_{[l / i]} \mathrm{s}_{[j / i]} x$.

Quasipolyadic algebra, QPA: [14]. 
A quasipolyadic algebra of dimension $n$, briefly a $\mathbf{Q P A}{ }_{n}$, is an algebra

$$
\mathfrak{A}=\left(A,+,-, \mathrm{c}_{i}, \mathrm{~s}_{[i / j]}, \mathrm{s}_{[i, j]}: i, j<n\right)
$$

where the reduct to $\mathbf{S c}_{n}$ is a substitution algebra (it satisfies (1)-(10) above) and additionally it satisfies the following equations for all $i, j, k<n$ :

$2^{\prime} \mathrm{s}_{[i / i]}(x)=\mathrm{s}_{[i, i]}(x)=x$, and $\mathrm{s}_{[i, j]}=\mathrm{s}_{[j, i]}$,

3 ' $\mathbf{s}_{[i / j]}$ and $\mathbf{s}_{[i, j]}$ are boolean endomorphisms

11. $\mathrm{s}_{[i, j]} \mathrm{S}_{[i, j]} x=x$,

12. $\mathrm{s}_{[i, j]} \mathrm{S}_{[i, k]}=\mathrm{s}_{[j, k]} \mathrm{S}_{[i, j]}$ if $|\{i, j, k\}|=3$,

13. $\mathrm{S}_{[i, j]} \mathrm{S}_{[j / i]} x=\mathrm{S}_{[i / j]} x$.

Quasipolyadic equality algebra, QPEA: [14].

A quasipolyadic equality algebra of dimension $n$, briefly a $\mathbf{Q P E \mathbf { A } _ { n }}$ is an algebra

$$
\mathfrak{B}=\left(\mathfrak{A}, \mathrm{d}_{i j}\right)_{i, j<n}
$$

where $\mathfrak{A}$ is a $\mathbf{Q P A} \mathbf{A}_{n}$ (i.e. it satisfies all the equations above), $\mathrm{d}_{i j}$ is a constant and the following equations hold, for all $i, j, k<n$ :

14. $\mathrm{s}_{[i / j]} \mathrm{d}_{i j}=1$,

15. $x \cdot \mathrm{d}_{i j} \leq \mathrm{s}_{[i / j]} x$.

\section{REFERENCES}

[1] T SAyed Ahmed and B SAmir, A neat embedding theorem for expansions of cylindric algebras, Logic Journal of IGPL, vol. 15 (2007), pp. 41-51.

[2] H Andréka, S Givant, S Mikulás, I NÉmeti, and A Simon, Notions of density that imply representability in algebraic logic, Ann. Pure. Appl. Logic, vol. 91 (1998), pp. 93-190.

[3] A Daigneault and J Monk, Representation theory for polyadic algebras, Fundamenta Math., vol. 52 (1963), pp. 151-176.

[4] L Henkin, J Monk, and A TARski, Cylindric algebras part i, North-Holland, 1971.

[5] — Cylindric algebras part ii, North-Holland, 1985.

[6] R Hirsch and I Hodkinson, Relation algebras from cylindric algebras, I, Ann. Pure. Appl. Logic, vol. 112 (2001), pp. 225-266.

[7] - Relation algebras from cylindric algebras, II, Ann. Pure. Appl. Logic, vol. 112 (2001), pp. 267-297.

[8] - Relation algebras by games, North-Holland, Elsevier Science, Amsterdam, 2002.

[9] R Hirsch, I Hodkinson, and R Maddux, Provability with finitely many variables, 2002.

[10] - Relation algebra reducts of cylindric algebras and an application to proof theory, J. Symbolic Logic, vol. 67 (2002), no. 1, pp. 197-213.

[11] I NÉmeti, The class of neat reducts is not a variety but is closed w.r.t HP, Notre Dame J. Formal Logic, vol. 24 (1983), pp. 399-409.

[12] - Algebraisations of quantifier logics, an introductory overview, Studia Logica, vol. 50 (1991), no. 3/4, pp. 485-570, An extended version of this paper is available at http:// math-inst.hu/pub/algebraic-logic/survey.dvi.

[13] C Pinter, A simple algebra of first order logic, Notre Dame Journal of Formal Logic, vol. 1 (1973), pp. 361-366.

[14] I SAIN and R Thompson, Strictly finite schema axiomatization of quasi-polyadic algebras, Algebraic logic (H Andréka, J Monk, and I Németi, editors), Colloq. Math. Soc. J. Bolyai, vol. 54, North-Holland, Amsterdam, 1991, pp. 539-571. 